\title{
Correction to: Computational investigation of single-wall carbon nanotube functionalized with palladium nanoclusters as hydrogen sulfide gas sensor
}

\author{
S. Bagherzadeh-Nobari ${ }^{1} \cdot$ K. Hosseini-Istadeh $^{2} \cdot$ R. Kalantarinejad ${ }^{3} \cdot$ S. M. Elahi ${ }^{1} \cdot$ A. A. Shokri ${ }^{4}$
}

Published online: 10 April 2018

(c) The Author(s) 2018

\section{Correction to: International Nano Letters https://doi.org/10.1007/s40089-018-0226-6}

The original version of this article unfortunately contained a mistake. The orientation of Fig. 1 was incorrect. The correct orientation is shown below.
Open Access This article is distributed under the terms of the Creative Commons Attribution 4.0 International License (http://creativeco mmons.org/licenses/by/4.0/), which permits unrestricted use, distribution, and reproduction in any medium, provided you give appropriate credit to the original author(s) and the source, provide a link to the Creative Commons license, and indicate if changes were made.

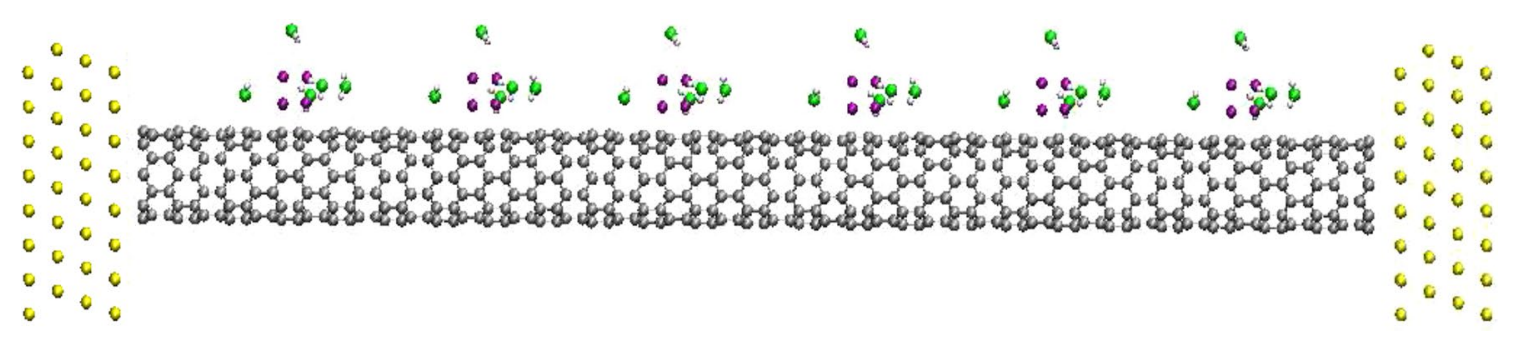

Fig. 1 Geometry of the conductometric $\mathrm{H}_{2} \mathrm{~S}$ sensor, based on carbon nanotube, decorated with Pd groups. Hydrogen atoms (white), sulfur atoms (green), gold electrodes (yellow), CNT (gray) and palladium clusters (purple)

The original article can be found online at https://doi.org/10.1007/ s40089-018-0226-6.

R. Kalantarinejad

kalantari@shezan.ir

$1 \quad$ Plasma Physics Research Center, Science and Research Branch, Islamic Azad University, Tehran, Iran

2 Shezan Research and Innovation Centre, No. 25, Innovation 2 St., Pardis Tech Park, Tehran, Iran

3 Aerospace Research Institute, Ministry of Science, Research and Technology, Tehran, Iran

4 Department of Physics, Payame Noor University (PNU), Tehran 19395-3697, Iran 